\title{
From Formal Concept Analysis to Contextual Logic
}

\author{
Frithjof Dau and Julia Klinger \\ Technische Universität Darmstadt, Fachbereich Mathematik \\ Schloßgartenstr. 7, D-64289 Darmstadt, \\ \{dau,jklinger\}@mathematik.tu-darmstadt.de
}

\begin{abstract}
A main goal of Formal Concept Analysis from its very beginning has been the support of rational communication. The source of this goal lies in our understanding of mathematics as a science which should encompass both its philosophical basis and its social consequences. This can be achieved by a process named 'restructuring'. This approach shall be extended to logic, which is based on the doctrines of concepts, judgments and conclusions. The program of restructuring logic is named Contextual Logic (CL). A main idea of CL is to combine Formal Concept Analysis and Concept Graphs (which are mathematical structures based on conceptual graphs). Concept graphs formulate judgments on the contained concepts, and conclusions can be drawn by inferring one concept graph from another. So we see that concept graphs can be understood as a crucial part of the mathematical implementation of CL, based on Formal Concept Analysis as the mathematization of the doctrine of concepts.
\end{abstract}

\section{Overview}

Formal Concept Analysis (FCA) is a mathematical theory applied successfully in various projects (there have been more than 200 projects in various academic and commercial fields). The impact and success of FCA and the large number of applications in the real world cannot be explained solely with the mathematical results and the mathematical power of FCA. The driving force behind FCA lies in our understanding of mathematics as a science which encompasses the philosophical basis and the social consequences of this discipline as well.

A main goal of Formal Concept Analysis from its very beginning has been the support of rational communication and the representation and processing of knowledge. This goal is based on and carried out by a process termed 'restructuring'. In the first section, we will describe the ideas and purposes of this restructuring process as well as further philosophical foundations of FCA. Moreover, we will argue why FCA fulfils the purposes of restructuring to a large extent.

In the next section, the restructuring approach is extended to logic. We will show that the purely extensional and mechanistic attempt of contemporary formal mathematical logic is too narrow for our purposes. For this reason, we revitalize the traditional philosophical understanding of logic. This understanding 
tries to capture and investigate the laws of thinking and is based on the doctrines of concepts, judgments and conclusions.

The program of restructuring mathematical logic will be called Contextual Logic (CL). As this logic starts with the doctrine of concepts, we see that CL builds upon FCA. However, further formalizations of the doctrines of judgments and conclusions are needed.

A promising approach is to use the system of conceptual graphs (CGs) by John Sowa, which will be introduced in Section 4. The philosophical background of this system is similar to that of FCA, and the system allows us to formulate judgments and conclusions in a way which is much nearer to human reasoning than predicate logic. But the system of conceptual graphs is not elaborated mathematically. One reason for this is that this system is very open-minded and extended in various ways which are often not clearly defined. Thus, it is huge, without sharp borders, and contains several ambiguities, gaps and even flaws and mistakes.

For this reason, in Section 5 a mathematical formalization for a core of CGs is provided. The mathematical structures modelling CGs will be called concept graphs. As said above, it is impossible to find a definition for concept graphs which covers all aspects and features of CGs at once. Instead of that, different versions of concept graphs which correspond to different fragments of CGs are elaborated. An overview over the different kinds of concept graphs is presented in Section 6.

\section{Formal Concept Analysis}

From the main theorem of Formal Concept Analysis we know that the concept lattices of FCA are -up to isomorphism- exactly the complete lattices of lattice theory. Thus, from a purely mathematical point of view, FCA can be seen as the theory of complete lattices, presented in an unfamiliar way. The broad results of lattice theory have been applied in various fields of mathematics, but only little outside mathematics. In contrast to that, FCA has been successfully applied in various projects. Thus, in order to explain the power and success of FCA, it is obviously not sufficient to look at FCA as a solely mathematical structure theory. The question is: What is the unique peculiarity that makes FCA that usable? What is the advantage of FCA compared to the usual form of lattice theory? The answer lies in the underlying philosophy of FCA. The main idea is a program termed 'restructuring (lattice theory)', relying on the concept of Wissenschaftsdidaktik by Hartmut von Hentig. In his book 'Magier oder Magister? Über die Einheit der Wissenschaft im Verständigungsprozess.' ('Magician or Magister? On the Unity of Science in the Process of Understanding', cf. [He74]), the restructuring of scientific disciplines is explained as follows:

Sciences have to examine their disciplinary, and this means: To uncover the unconscious purposes, to declare their conscious purposes, to select and to adjust their means according to those purposes, to explain possible consequences comprehensible and publicly, and to make accessible 
their ways of scientific finding and their results by the every-day language. (p. 136f)

Hentig, 1974

The program of restructuring is based on a philosophical background which goes back to the Pragmatism of Charles Sanders Peirce (see [Pe35]) and which is adopted and continued in the Discourse Philosophy of Karl-Otto Apel (cf. [Ap89]) and Jürgen Habermas (see [Ha81]). The main idea of Pragmatism is that the significance of any conception consists exclusively in its effects. In particular, each scientific concept and theory has to be judged by all the effects it may produce. This establishes a tight connection between theory and practice.

Another crucial point is that in Pragmatism and discourse philosophy, the basis and origin of reasoning lies within intersubjective communication and argumentation. It is important to note that intersubjective communication takes place not only between members of a specific scientific community, but between members of different communities and even between scientists and non-scientists. Thus, a transdisciplinary communication has to be enabled and established. For this reason, Hentig demands the use of every-day language, so that a scientific theory, including its results and effects, can be understood, applied and critizised by people standing outside that specific scientific community. In another place in [He74] he says:

The restructuring of scientific disciplines within themselves becomes more and more necessary to make them more learnable, mutually available, and criticizable in more general surroundings, also beyond disciplinary competence. This restructuring may and must be performed by general patterns of perception, thought, and action of our civilization. (p. 33f)

$$
\text { Hentig, } 1974 \text { (italics by Dau/Klinger) }
$$

The development of FCA is grounded on Hentings restructuring program. Lattice theory is reworked in order to integrate and to rationalize origins, connections to and interpretations in the real world. The results of lattice theory have to be presented in a way which makes them understandable, learnable, available and criticizable, particularly for non-mathematicians. As Wille says in [Wi96]:

The aim is to reach a structured theory which unfolds the formal thoughts according to meaningful interpretations allowing a broad communication and critical discussion of the content.

Wille, 1996

We have to discuss why and how FCA achieves the requirements of the restructuring program. The starting point of FCA is the philosophical understanding of a concept as the basic unit of thought. A concept is constituted by two counterparts: Its extension which consists of all objects belonging to the concept, and its intension which contains all attributes shared by all objects of the extension. Due to Peirce, in any reasoning or argumentation process, we can only grasp a limited part of the reality. Our universe of discourse is always a restricted context. These 
considerations lead to the well-known basic definitions of FCA which formalize these ideas. We start with a formal context $(G, M, I)$, consisting of a set $G$ of (formal) objects (in German: 'Gegenstände'), a set $M$ of (formal) attributes (in German: 'Merkmale'), and an incidence-relation $I \subseteq G \times M$. The relationship $g I m$ (with $g \in G$ and $m \in M$ ) indicates that the object $g$ has the attribute $m$. A formal concept is a pair $(A, B)$ with $A \subseteq G$ and $B \subseteq M$, which satisfies $B=\{m \in M \mid g I m$ for all $g \in A\}$ and $A=\{g \in G \mid g I m$ for all $m \in B\}$. This is clearly a mathematical formalization of the philosophical concepts.

From a mathematical point of view, formal contexts and formal concepts could be reduced to classical relational structures (which are purely extensional) resp. to unary predicates. But humans structure the world conceptually and meaningfully, and the meaning of concepts cannot be explained solely by their extensions. On the contrary: The meaning of concepts is heavily constituted by their intensions and by the intermediate relationships between the concepts (cf. [Se01] and [Br94]). Moreover, a formal context can be represented by crosstables which are very common in our culture and therefore easy to comprehend. Thus, from a human point of view, formal contexts are easier to understand and much more meaningful than relational structures.

The most important relation between concepts is given by the relationships subconcept and superconcept. For formal concepts, they are defined as follows: Given two formal concepts $\left(A_{1}, B_{1}\right)$ and $\left(A_{2}, B_{2}\right)$, we set $\left(A_{1}, B_{1}\right) \leq$ $\left(A_{2}, B_{2}\right): \Longleftrightarrow A_{1} \subseteq A_{2}\left(\Longleftrightarrow B_{2} \subseteq B_{1}\right)$ and say that $\left(A_{1}, B_{1}\right)$ is a subconcept of $\left(A_{2}, B_{2}\right)$ resp. $\left(A_{2}, B_{2}\right)$ is a superconcept of $\left(A_{1}, B_{1}\right)$. The set of all formal concepts of a given formal context, together with the relation $\leq$, is a complete lattice, the concept lattice of the formal context.

The concept lattice of a formal context can be represented as a labelled line diagram. This visualization of the underlying formal context is the next advantage of FCA. With a small amount of experience, these diagrams are easy to understand and comprehend. Our experience with projects shows that when a specific domain is formalized by a formal context, the contemplation of the line diagram of the corresponding concept lattice often leads to unexpected insights by the domain experts.

Still more effects come into play when so-called many-valued contexts are considered. Many-valued contexts are transformed into ordinary (one-valued) formal contexts by the process of conceptual scaling. During this process, each attribute $m \in M$, together with its values, is interpreted by a context itself. There is no standard or even neccessary interpretation of an attribute: It has to be decided by the field expert which scale is appropriate for a given attribute. The fact that the process of scaling cannot be automated should be understood not as a drawback, but a great advantage: In our projects it turned out, when a ToscAnA-System ${ }^{1}$ was implemented by ToscANA-consultants (which are experts on FCA, but which usually have no or only few experience in the specific domain), that the right choice of a scale is usually far from being trivial and often

\footnotetext{
${ }^{1}$ ToscAnA is a computer program which allows to explore relational databases with FCA-methods, see for instance [BH03].
} 
raises discussions among the field experts as well as between the field experts and the ToscAnA-consultants. Thus, already the process of scaling supports rational communication, even between members of different research fields. Moreover, as the choice of the scales is left to the experts and not done automatically by the machine, the cognitive autonomy and the responsibility of the experts is preserved.

Finally, many-valued contexts can be understood as mathematical implementations of tables in relational databases. As most information is stored as data in relational databases, FCA turns out to be a perfect instrument for a meaningful and conceptual exploration of the stored data.

\section{Logic}

The aim of restructuring logic seems to be self-evident, as logic, understood as the investigation of the laws of thinking, is another fundamental source for reasoning. Due to the pragmatic paradigm, the first step in the restructuring process has to make clear the purposes and effects of logic.

In the previous section, it was already argued that the purely extensional approach to predicate logic and their models (relational structures) is to narrow to be used in rational and meaningful discourses. As another point, the mechanistic attempt of predicate logic shall be mentioned, particulary the calculi which act on formulas, understood as a priori meaningless sequences of signs. The reason for this is clear: The purpose of predicate logic has never been to model or support human reasoning, but to provide an instrument which shall explain and contribute to the structure of mathematical argumentations only. Thus, for supporting reasoning, we have to find a broader understanding of logic which goes beyond classical predicate logic. As Apel says in [Ap89]:

In view of this problematic situation [of rational argumentation] it is more obvious not to give up reasoning at all, but to break with the concept of reasoning which is orientated by the pattern of logic-mathematical proofs.

$$
\text { Apel, } 1989 \text { (italics by Dau/Klinger) }
$$

A convincing approach is to revitalize the traditional, philosophical understanding of logic, given by 'the three essential main functions of thinking - concepts, judgments and conclusions' [Ka88]. Concepts, the basic units of thought, are already formalized in FCA. If we combine concepts to meaningful statements, we obtain propositions; and judgments are propositions which are asserted, i. e. valid propositions (due to Peirces pragmatism and the discourse philosphy, the validity of judgments has to be confirmed by a rational discourse in the intersubjective community of communication). With conclusions, new judgments are obtained from already existing ones.

The process of restructuring this understanding of logic, i. e. the mathematical formalization of philosophical logic shall lead to a theory called Contextual Logic (CL). In CL, we have to formalize concepts, judgments, and conclusions. 
As the mathematization of concepts has already been elaborated, the question of how to proceed with judgments and conclusions arises.

\section{Conceptual Graphs}

For the formalization of judgments, we use the theory of Conceptual Graphs $(C G)$ by John Sowa. In Figure 1, we provide two well-known examples of CGs.

CAT: Yoyo on MAT:*

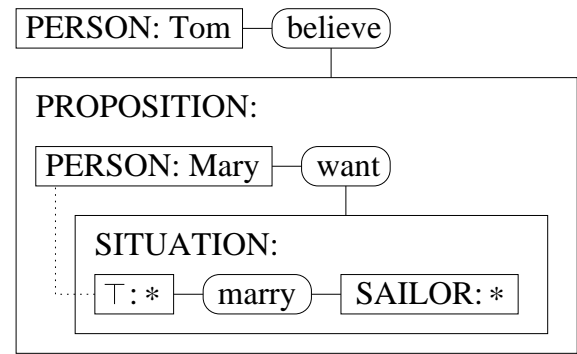

Fig. 1. Two conceptual graphs

Conceptual graphs are assembled of concept boxes, each of them containing a type and a reference belonging to the type. The star in the right concept box of the left graph is the so-called generic marker and has to be understood as an unqualified object; i.e. the generic marker can be read as an existential quantifier. Concept boxes may be nested (see the right graph for an example). In this case, a box stands for a context, and the reference of the box is a graph itself which describes this context. Concept boxes may be connected by relation ovals or by a dotted line (so-called coreference-links). These connections describe relationships between the references of the boxes. In particular, a coreference-link between two concept boxes means that their references are identical.

The meaning of the left graph is therefore 'The cat Yoyo is on a mat'. The right graph contains two contexts, namely the concept boxes of type PROPOSITION and SITUATION (which are common types of contexts). The graph can be read as follows: The person Tom believes a proposition, which is described by a graph itself. The proposition says that the person Mary wants a situation, which again is described by a graph. In this situation we have a concept box $\top: *$ connected with a coreference link to the concept box PERSON:Mary in the context above. So the situation is that Mary marries a sailor. The understanding of the whole graph is now: The person Tom believes the proposition that the person Mary wants the situation that Mary marries a sailor. In short: Tom believes that Mary wants to marry a sailor.

In [So92], Sowa explains the foundations and the purpose of conceptual graphs as follows: 
Conceptual graphs are a system of logic based on the existential graphs of Charles Sanders Peirce and the semantic networks of artificial intelligence. The purpose of the system is to express meaning in a form that is logically precise, humanly readable, and computationally tractable. With their direct mapping to language, conceptual graphs can serve as an intermediate language for translating computer-oriented formalisms to and from natural languages. With their graphic representation, they can serve as a readable, but formal design and specification language.

Sowa, 1992

As this quotation shows, the philosophy behind conceptual graphs is based on Peirce' pragmatism, and it is very close to Hentigs restructuring program.

In philosophy, the considered judgments are often elementary, i. e. judgments of the form 'an object belongs to the extension of a concept' or 'one concept is a subconcept of another concept'. The first kind of judgments corresponds to the boxes in conceptual graphs, the second kind is coded in the so-called type hierarchy of conceptual graphs. But as we can see in Figure 1, with conceptual graphs, much more complex judgments can be formulated. This higher expressiveness is clearly a further advantage.

Sowa provides rules for formal deduction procedures on conceptual graphs. Thus the system of conceptual graphs offers a formalization of conclusions as well.

In some sense the system of conceptual graphs is not fixed, but open-minded. It is designed to be used in fields like software specification and modelling, knowledge representation, natural language generation and information extraction, and these fields have to cope with problems of implementational, mathematical, linguistic or even philosophical nature. In order to deal with such problems, different modifications and extensions of conceptual graphs are suggested. For this reason it is impossible (and perhaps not even desirable) to provide a definition which covers all possible aspects and features of conceptual graphs at once. On the other hand a closer observation shows that this leads to a lack of preciseness which causes several difficulties and fallacies, ranging from ambiguities over minor gaps to major flaws.

As Sowas system is not mathematically elaborated, we have to provide mathematical definitions for conceptual graphs, and we have to formalize how reasoning can be carried out with them. An evident approach is to interpret the types of conceptual graphs by formal concepts. But as argued above, it cannot be expected to find a mathematical definition which covers all aspects of conceptual graphs at once. Instead of this, different forms of concept graphs (as a mathematization of CGs) with different levels of expressiveness (and further differences) have been developed during the last years. An overview of the different forms of concept graphs will be provided in the next sections. 


\section{Concept Graphs and Contextual Logic}

As explained in the previous sections, Contextual Logic can be understood as a formalization of the traditional philosophical logic with its doctrines concepts, judgments and conclusions. In this section we explain briefly how the theory of CGs combined with FCA led to the theory of concept graphs in its several distinct formings.

Concept graphs are based on CGs. However, as elaborated in the previous section, CGs were designed to be of use in a wide variety of different fields, resulting in difficulties in the mathematization. Hence, concept graphs as mathematization of CGs only cover restricted parts of Sowa's Theory. In general, two accesses can be distinguished, namely semantical approaches and those based on a separation in syntax and semantics.

Semantical theories deal with the elaboration of a mathematical structure theory of concept graphs of a given power context family in an algebraic manner (where a power context family (PCF) is a family of contexts which are connected via their object sets; the mathematical definition can be found below). In particular, the forms and relations of those concept graphs are investigated. This includes operations on those graphs (see for instance [Wi01]) and a thorough study of the properties of the corresponding algebra of concept graphs of a given power context family. Since semantical approaches to the theory of concept graphs are concerned with all 'valid propositions' of a power context family, they are understood as a formalization of the doctrine of judgments.

The other approaches are logical ones, using a separation of syntax and semantics as it is common in mathematical logic: Concept graphs as syntactical constructs are defined over an alphabet consisting of object-, concept- and relation-names. They are then equipped with an explicit contextual semantics based on power context families (instead of the traditional implicit semantics of CGs via a translation into predicate logic). In many of the approaches, the results of the corresponding semantical access are used. Since the different specificities of logic systems of concept graphs include an adequate calculus (i. e. a set of inference rules which are sound and complete) or (for the theories of Prediger and Klinger) an effective method to do reasoning via standard models/PCFs, these theories are considered to be a formalization of the doctrine of conclusions.

We will now briefly explain the basic notions of those two approaches, starting with concept graphs as semantical structures.

The first approach to Contextual Judgment Logic was proposed by Wille in [Wi97] where he connected FCA with the theory of CGs. Wille defined concept graphs of power context families as semantical structures.

Since this first access was further developed and specified, we will use a slightly more recent version of concept graphs. The following definitions are taken from [Wi00b]: A power context family $\overrightarrow{\mathbb{K}}:=\left(\mathbb{K}_{0}, \mathbb{K}_{1}, \mathbb{K}_{2}, \ldots, \mathbb{K}_{n}\right)$ is a family of contexts $\mathbb{K}_{k}:=\left(G_{k}, M_{k}, I_{k}\right)$ with $G_{0} \neq \emptyset$ and $G_{k} \subseteq\left(G_{0}\right)^{k}$ for each $k=1, \ldots, n$. The formal concepts of $\mathbb{K}_{k}$ with $k=1, \ldots, n$ are called relation concepts because they represent $k$-ary relations on the object set $G_{0}$ by their extent. A concept graph is defined as a structure $\mathfrak{G}:=(V, E, \nu, \kappa, \rho)$ consisting of two sets $V$ and $E$ 
and three functions $\nu, \kappa$, and $\rho$ such that the following conditions are satisfied: Firstly, $(V, E, \nu)$ has to be a relational graph consisting of two disjoint sets $V$ and $E$ whose elements are called vertices and edges, respectively, and a function $\nu: E \rightarrow \bigcup_{k=1, \ldots, n} V^{k}$ which maps each edge to the ordered tuple of its adjacent vertices. Secondly, $\kappa: V \cup E \rightarrow \bigcup_{k=0, \ldots, n} \underline{\mathfrak{B}}\left(\mathbb{K}_{k}\right)$ is a mapping such that $\kappa(u) \in$ $\underline{\mathfrak{B}}\left(\mathbb{K}_{k}\right)$ for all $u \in V \cup E$ with $|u|=k$. Finally, $\rho: V \cup E \rightarrow \bigcup_{k=0, \ldots, n} \mathfrak{P}\left(G_{k}\right) \backslash\{\emptyset\}$ is a mapping such that $\rho(u) \subseteq \operatorname{Ext}(\kappa(u))$ for all $u \in V \cup E$ and, furthermore, $\rho(u)=\rho\left(v_{1}\right) \times \cdots \times \rho\left(v_{k}\right)$ if $|u|=k>0$ and $\nu(u)=\left(v_{1}, \ldots, v_{k}\right)$.

An order on the set of all concept graphs of a power context family is then introduced via the so-called conceptual content, which is studied extensively in [Wi03]. It is even shown that the conceptual contents of concept graphs of a power context family can be described as extents of concepts of a suitably constructed power context family again.

As an example, we consider the different theories of concept graphs with papers they were published in, the kind of approach which was taken and the relation 'is an extension of' between these theories. The first graph of Figure 4, for instance, is a semantical concept graph of the PCF shown in Figure 2. It is read as follows: Triadic Concept Graphs in [Wi98] have a semantic approach, Concept Graphs in [Wi97] as well and the theory presented in [Wi98] extends [Wi97]. Note that only one possible diagrammatic representation for the corresponding mathematical entity is shown in Figure 4.1.

$\mathbb{K}_{0}:$

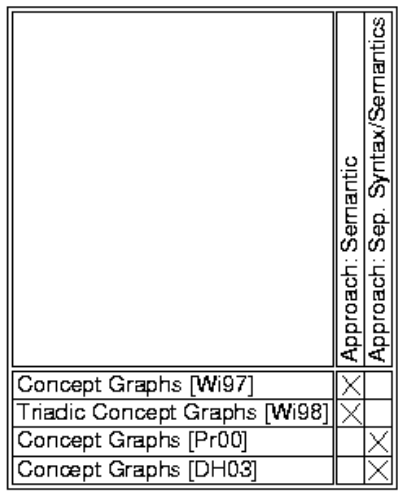

$\mathbb{K}_{2}$ :

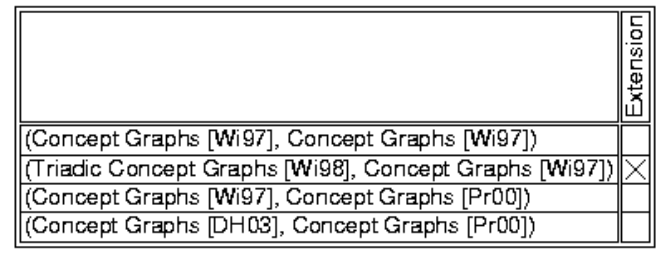

Fig. 2. The power context family $\left(\mathbb{K}_{0}, \mathbb{K}_{2}\right)$

As an approach based on the seperation of syntax and semantics, we describe how concept graphs were introduced in [Pr98b], where the approach of [Wi97] was adopted and modified in order to obtain a logical theory:

The first step towards a syntactical implementation of concept graphs is the definition of an alphabet $\mathcal{A}:=(\mathcal{G}, \mathcal{C}, \mathcal{R})$ consisting of a set $\mathcal{G}$ of object names, 


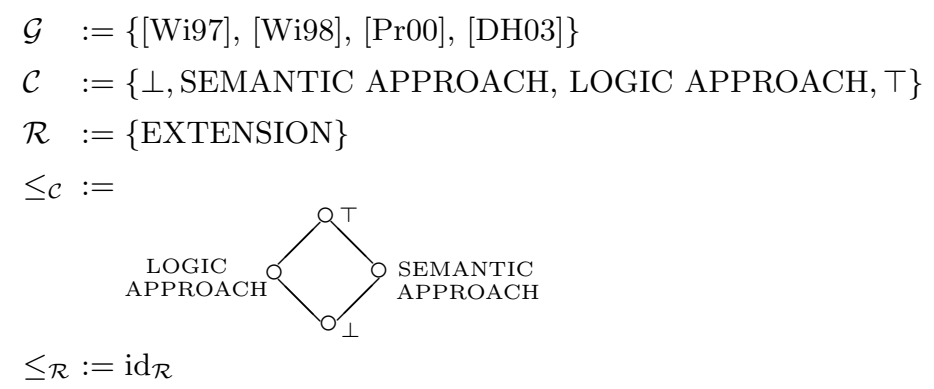

Fig. 3. Example for an Alphabet

an ordered set $\mathcal{C}$ of concept names and a family $\mathcal{R}$ of ordered sets of relation names. An example for such an alphabet is given in Figure 3. To distinguish the syntactical names from the elements of the power context family used for the interpretation, we employ different capitalizations. such an alphabet is given. Syntactical concept graphs over an alphabet are then introduced as mathematical structures of the form $\mathfrak{G}:=(V, E, \nu, \kappa, \rho)$, consisting of a relational graph, a function $\kappa$ assigning concept names to vertices and relation names to edges, and a function $\rho$ which assigns non-empty sets of object names to the vertices (as references). A syntactical concept graph over the alphabet in Figure 3 is shown in Figure 4.2. syntactical concept graph over the alphabet depicted 3.

For the semantics, the names of the alphabet are interpreted in a given power context family $\overrightarrow{\mathbb{K}}:=\left(\mathbb{K}_{0}, \mathbb{K}_{1}, \ldots, \mathbb{K}_{n}\right)$ via a so-called interpretation $\lambda$ : This interpretation specifies how the syntactical elements of the alphabet are related to elements of $\overrightarrow{\mathbb{K}}$ such that object names are mapped to objects of $\mathbb{K}_{0}$, concept names to concepts of $\mathbb{K}_{0}$ and relation names to elements of $\mathfrak{B}\left(\mathbb{K}_{k}\right)$. Moreover, the orders specified in the alphabet are preserved. The resulting structures $(\overrightarrow{\mathbb{K}}, \lambda)$ are called context-interpretations. Now we say that a concept graph is valid in a context-interpretation $(\overrightarrow{\mathbb{K}}, \lambda)$ (and call $(\overrightarrow{\mathbb{K}}, \lambda)$ a model for the graph) if the so-called vertex- and edge condition for the vertices respectively edges are both satisfied. The vertex condition for a vertex $v$ is fulfilled if the interpreted object names of $v$ belong to the extent of the interpreted concept name of that vertex. Similarly, the edge condition for an edge $e$ holds if the objects along $e$ are in the relation concept assigned to that edge. It is easy to see that this graph is (using a suitable interpretation) indeed a concept graph of the PCF in Figure 2.

Why does the theory of concept graphs meet the claims for Contextual Logic?

The aim of Contextual Logic is not to enter into competition with First Order Predicate Logic (FOPL), in particular since the expressiveness of most of the theories corresponds only to restricted parts of FOPL. The goal was rather to find an approach which may support rational communication and argumentation. In contrast to FOPL, CGs have been developed and used as a language for knowledge representation with its focus on connections of concepts. They aim at capturing the rhetoric structure of common language and are graphically repre- 
1. Semantic Concept Graph:

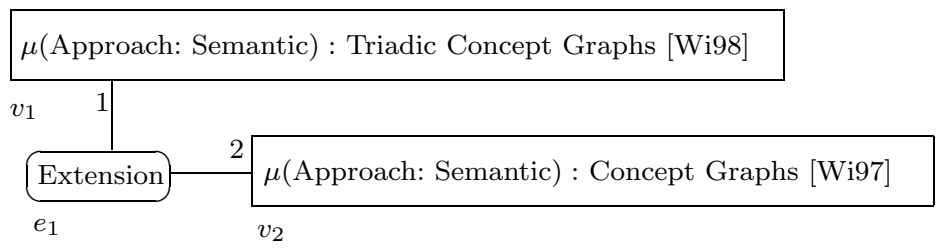

2. Syntactic Concept Graph:

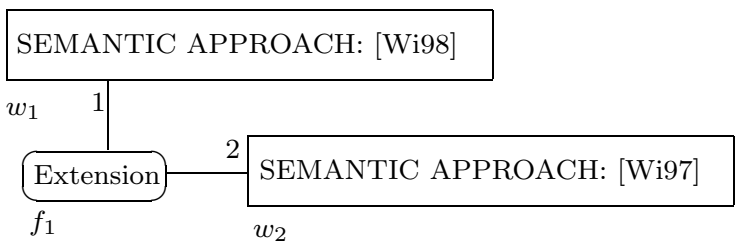

3. Syntactic Semiconcept Graph:

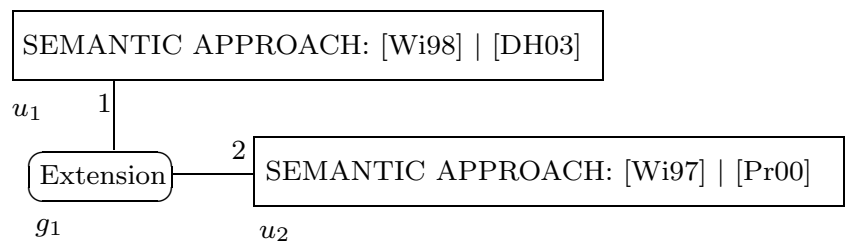

4. Syntactic Concept Graph with Cuts:
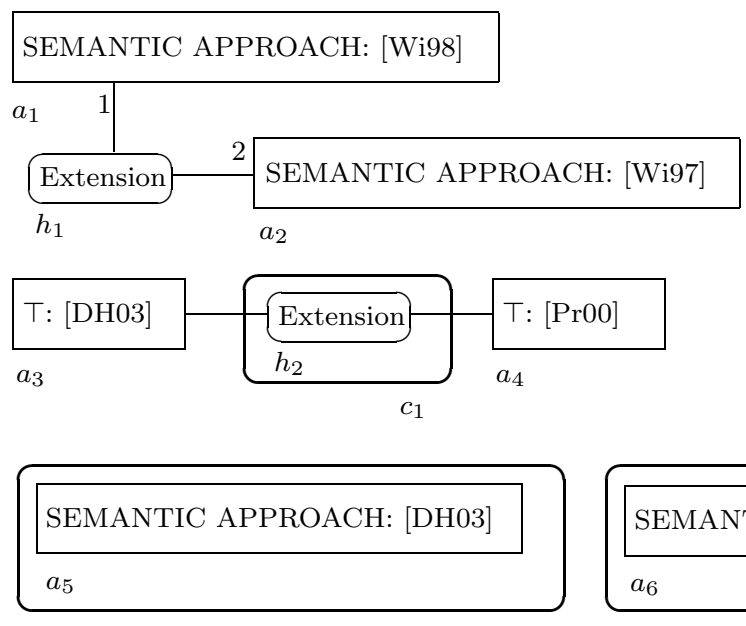

SEMANTIC APPROACH: [PrO0]

$a_{6}$

$c_{2}$

$c_{3}$

Fig. 4. Examples for Concept Graphs 
sentable. The contextual foundation of concept graphs as the mathematization of CGs enables us to make the restrictions which occur during the transition from real to formal data explicit and hence discussable. The various extensions for the basic theory of concept graphs as proposed by Wille in [Wi97] and developed by Prediger in [Pr98a], [Pr98b], yield a broad expressiveness for Contextual Logic. These extensions include negation on the level of propositions ([Da01], [Da02]), negation on the level of concepts and relations ([Wi01],[Wi02a], [Kl01a],[Kl01b]), existential ([Da01],[Wi02a], [Kl02]) and universal quantifiers ([Ta00]), and Nestings ([Wi98], [Pr00], [SW03], [DH03]). Moreover, the Contextual Logic of Relations has been developed ([PoW00], [Wi00c], [Ar02]) as a Contextual Attribute Logic $([\mathrm{GW} 99 \mathrm{~b}])$ on the relational contexts of a power context family and socalled relation graphs have been introduced as algebraic structures for representing relations and operations on relations ([Po01],[Po02]). In the next section we will discuss these extensions in more detail.

\section{Different Forms of Concept Graphs}

In this section, our aim is to give an overview over the characteristics and the diverse states of development of the different theories of concept graphs. Please note that the notion of 'concept graphs' is used as a generic term for all these approaches.

In Figure 5-7, several concept lattices are shown, which all have the same object set consisting of theories of concept graphs along with a reference for the corresponding paper. In each lattice, the attributes are chosen with respect to a certain focus (e.g. 'logical properties'). We will explain the attributes and give examples for the objects. Since the most fundamental differentiation for theories of concept graphs is that in semantic approaches and those established by a separation in syntax in semantics, this attribute occurs in each of the concept lattices.

The concept lattice in Figure $\mathbf{5}$ is about models and the kind of concepts which are considered (e.g. concepts, semiconcepts or protoconcepts for PCFs) for each semantical and syntactical theory in question.

All approaches have a so-called contextual semantics, which is based on FCA and describes concepts as the constituents of concept graphs in a formal and comprehensive way. The components of such a contextual model are specific kinds of formal contexts (depending on the particular system of concept graphs) and concepts of these contexts. Several models are distinguished: those based on power context families (PCFs) which are comprised by both triadic and nested PCFs, and relational contexts. For PCFs, there are three notions of concepts: The set of all protoconcepts of a context (see [Wi00a]) contains all semiconcepts which in turn include all concepts. Obviously, triadic PCFs lead to triadic concepts, and for nested PCFs and relational contexts, normal concepts are considered. In order to include, for instance, a negation on the level of concepts and relations, power context families with semiconcepts (or protoconcepts) can be employed as model since they include a negation on concepts. 


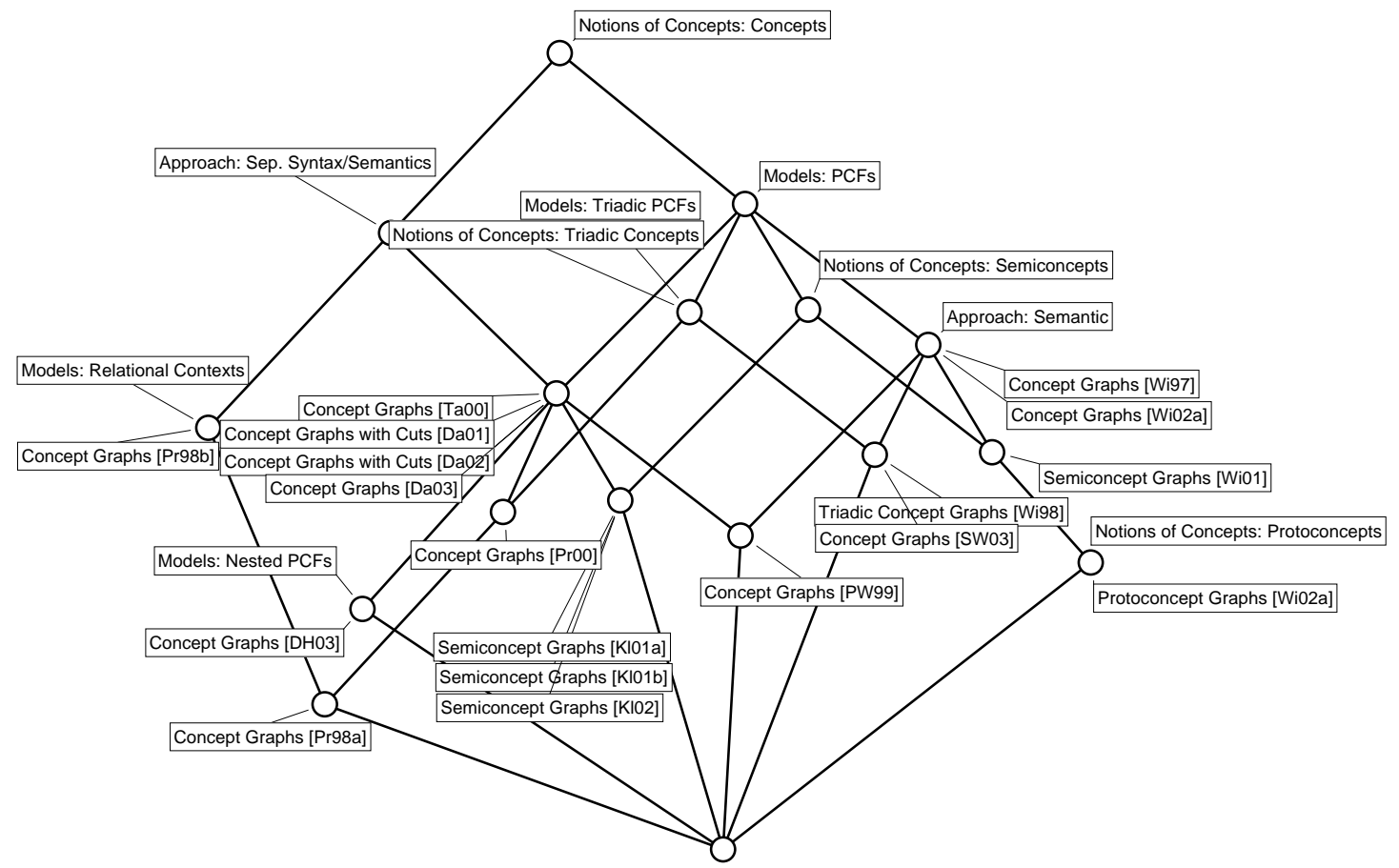

Fig. 5. Models and Units

The concept lattice shows, for instance, that relational contexts as models were only utilized by Prediger in [Pr98a] and [Pr98b]. They were then substituted by PCFs which yield a richer description of relations.

Figure 6 is concerned with extensions to the basic theory of concept graphs. There are several extensions and features, including the number of references, negation, nestings and quantification. We will now briefly summarize the meaning of the different attributes.

First negation is considered, which can be introduced on different levels. If the traditional philosophical understanding is taken into account, then (interpreted) concept graphs as judgments are regarded as valid propositions, hence as meaningful combinations of concepts. In particular, this means that the definition should not allow the construction of a self-contradictory graph. In order to still be in the position to employ a (though restricted) negation, in [Wi01] a negation on the level of concepts and relations was introduced. For the theory of concept graphs with cuts ([Da01]), the focus is different: Here the aim is to reach a logical equivalence to FOPL, thus the introduction of a negation on the level 


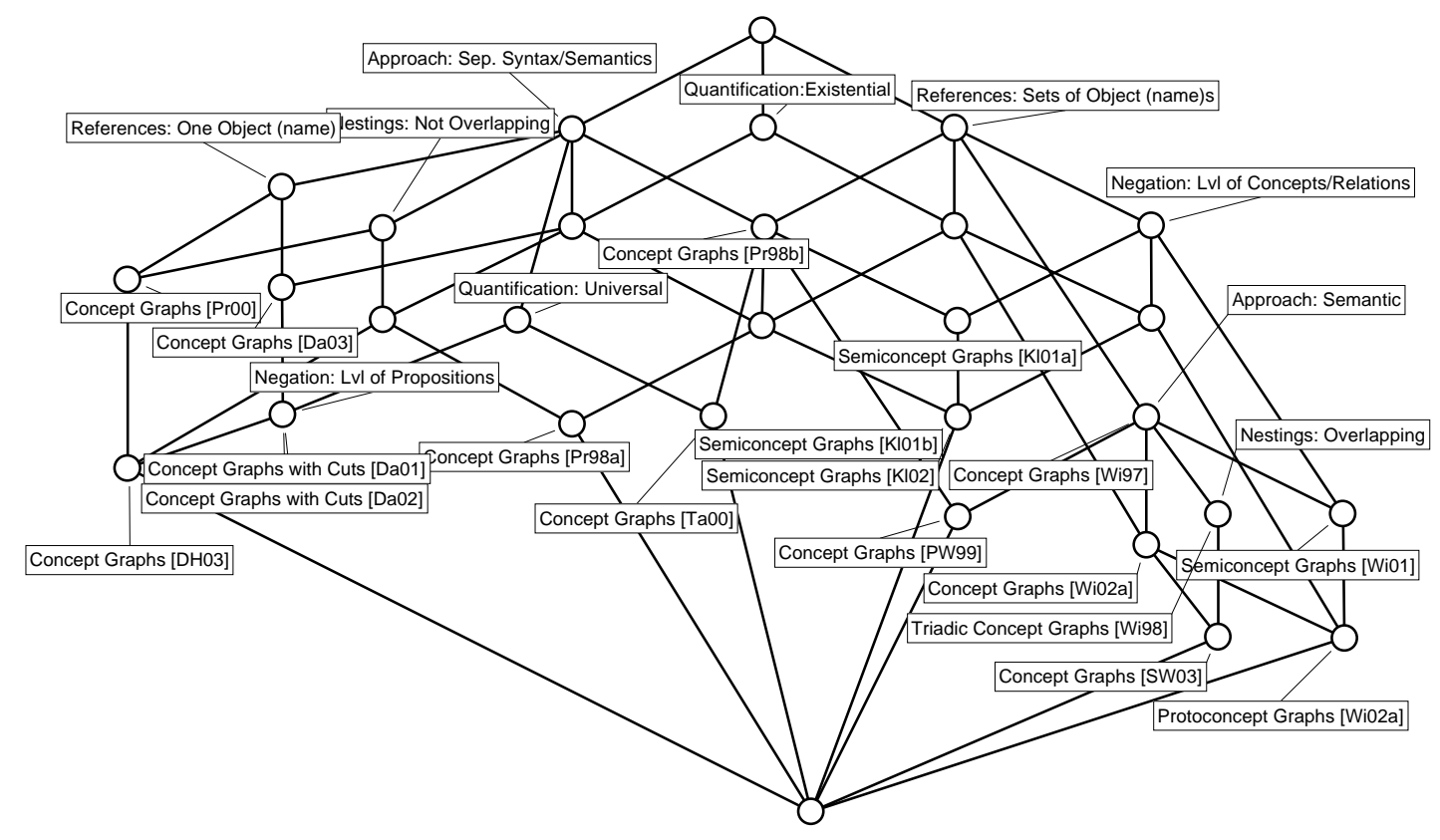

Fig. 6. Extensions and Features

of propositions is necessary. Moreover, as we have self-contradictory formulas in FOPL, it is allowed to construct self-contradictory graphs.

Two attributes are concerned with references, which are the objects (or, in logic approaches, the object names) assigned to the vertices of the relational graph. In some approaches there may be sets of objects assigned to each vertex, while other approaches only allow single objects. These first approaches therefore allow a more condensed representation of knowledge than the latter.

As for quantifiers, we find that there are two kinds of quantification for concept graphs. With existential quantification, existentially quantified variables are introduced as references, whereas universal quantification allows the assertion of propositions about all objects satisfying certain conditions. There is only one paper ([Ta00]) where universal quantifiers are introduced directly. In all other cases the topic of the corresponding paper is a system of concept graphs with existential quantification and global negation.

Finally we consider nestings (or subdivisions). Nestings form an extension to the language of concept graphs, which allows the bundling of information and the assertion of propositions which refer to different contexts (thus the coding of 'modal information'). There are theories which only consider disjoint nestings and those in which parts of the subdivision may overlap. 


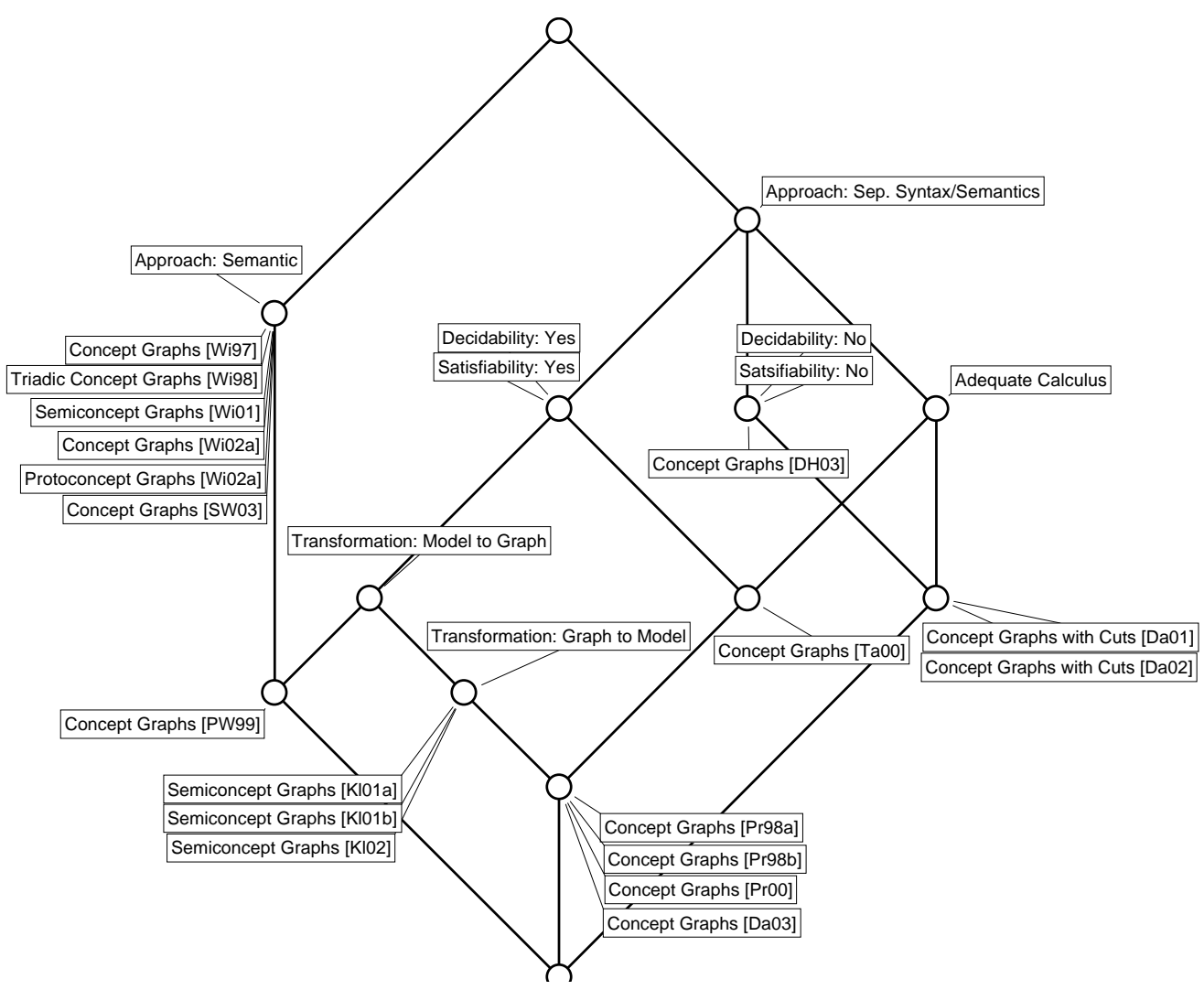

Fig. 7. Properties of Logical Theories

The concept lattice shows that all semantic theories have sets of objects as references, and, conversely, that only in the papers of Dau and one paper of Prediger the references are restricted to single objects. Moreover, by combining Figure 5 and 6 we can observe that all theories including nestings/subdivisions (which would comprise [Wi98] and [SW03] as semantic approaches and [Pr98a], $[\mathrm{Pr} 00]$ and [DH03] as logical ones) require PCFs with additional structures, such as triadic or nested PCFs.

Finally, Figure 7 addresses properties of logical theories. Again, we first outline the definitions of the attributes.

A logic system of concept graphs is called satisfiable if each syntactical concept graph is valid in at least one model, thus if the construction of selfcontradictory concept graphs is not possible. Moreover, in accordance with [Ba77], we say that a theory based on the separation of syntax and semantics is decidable, if there is an algorithm which determines for each pair $\mathfrak{G}_{1}, \mathfrak{G}_{2}$ of concept 
graphs over the same alphabet whether $\mathfrak{G}_{1}$ entails $\mathfrak{G}_{2}$ (hence if $\mathfrak{G}_{2}$ is valid in every model for $\mathfrak{G}_{1}$ ) or not.

Since the previous two attributes do not make sense for semantical approaches, we chose to state explicitly whether a logic approach satisfies them or not.

As for the remaining attributes: A logic system is said to have an adequate calculus, if there exists a sound and complete set of inference rules. Furthermore, we say that it is possible to transform a model into a concept graph, if for a given model (in particular, for a given PCF), a concept graph can be constructed which codes the same information as the PCF. This so-called standard graph then entails all other (valid) concept graphs of the corresponding model. Finally, the attribute Transformation: Graph to Model denotes that for each syntactical concept graph of the logic system in question it is possible to construct a model (or simply a power context family) coding the same information. Via this socalled standard model, entailment can be characterized.

In the concept lattice we can now see that the attribute 'Transformation: Graph to Model' implies both decidability and satisfiability. Satisfiability follows since in each of the corresponding papers the 'standard model' is indeed a model for the concept graph. Furthermore, in every papers having this attribute the entailment relation is characterized via the standard model or the standard PCF, hence the attribute 'Decidability: Yes' is implied, too.

Figure 4 shows examples for the different systems of concept graphs. The first two graphs were already explained in the previous section. The third graph is a (syntactical) semiconcept graph over the alphabet in Figure 3 and includes a negation on the concept and the relation level. It represents that the semantic approach in [Wi98] is an extension of the semantic approach described in [Wi97], that both [DH03] and [Pr00] are not semantic approaches, and that [DH03] is not a generalization of [Pr00] (which is interesting since both approaches deal with nestings, and [DH03] was published three years after [Pr00]). Hence, the references on the right side of the stroke are negative references with respect to both the corresponding concepts and relations. The last graph of Figure 4 is a concept graph with cuts ( assembled of three contiguous subgraphs) representing the same information as the third graph. As already mentioned, these graphs include a negation on the level of propositions. Informally speaking, a part of the graph is negated if it is nested in a so-called cut, which is represented in the Figure by a bold oval. The subgraphs not containing cuts are read in the same way as concept graphs. However, each cut negates everything within it, so the two bottommost parts of the graph are read: [DH03] and [Pr00] are 'T', [DH03] is not an extension of [Pr00], and neither [DH03] nor [Pr00] are semantic approaches. Concept Graphs with nestings are omitted in this example, since a triadic (or nested) PCF would be required to provide a reasonable explanation.

For an overview of the theories of Prediger, Dau and Klinger with a more formal focus we refer to [KV03]. 


\section{Outlook}

The restructuring process of logic is still in its early stages. Some syntactical approaches still lack adequate calculi. Much effort has to be spent on further extensions of concept graphs in order to model further aspects of reasoning and communication. This will include various forms of background knowledge, like material implications as they are described in the book of Brandom ([Br01]). Furthermore, modal and contextual reasoning or different kinds of quantification have to be incorporated.

On the other hand, a restructured logic has to prove itself in practice. Thus, it is desirable to implement concept graphs, for example as computer programs, and to test out their usability in real life projects. Only projects can show whether our formalizations of logic are adequate for our purpose.

It will certainly be a long way to carry out all these steps. But as our experience with FCA shows, we can be quite optimistic that these goals can be reached in the long run.

\section{References}

[Ap89] K.-O. Apel: Begründung. In H. Seifert, G. Radnitzky (Eds.): Handlexikon der Wissenschaftstheorie. Ehrenwirth, München 1989, 14-19.

[Ar02] M. Arnold: Einführung in die Relationenlogik. Diplomarbeit. FB Mathematik, TU Darmstadt 2002.

[Ba77] J. Barwise (Ed.): Handbook of Mathematical Logic. North-Holland Publishing Company, Amsterdam-New York-Oxford 1977.

[BH03] P. Becker, J. Hereth Correia: The ToscanaJ Suite for Implementing Conceptual Information Systems. This volume.

[Br94] R.B. Brandom: Making it explicit. Reasoning, Representing, and Discursive Commitment. Harvard University Press, Cambridge 1994.

[Br01] R.B. Brandom: Begründen und Begreifen. Eine Einführung in den Inferentialismus. Suhrkamp 2001.

[Da01] F. Dau: Concept Graphs and Predicate Logic. In: H. S. Delugach, G. Stumme (Eds.): Conceptual Structures: Broadening the Base, Springer Verlag, Berlin-New York 2001, 72-86.

[Da02] F. Dau: The Logic System of Concept Graphs with Negations (and its Relationship to Predicate Logic), Dissertation. FB Mathematik, TU Darmstadt 2002. For the submitted version see http://www. mathematik.tu-darmstadt.de/ dau

[Da03] F. Dau: Concept Graphs without Negations: Standardmodels and Standardgraphs. FB4-Preprint, TU Darmstadt 2003.

[DH03] F. Dau, J. Hereth Correia: Nested Concept Graphs: Mathematical Foundations and Applications in Databases. FB4-Preprint, TU Darmstadt 2003.

[GW99a] B. Ganter, R. Wille: Formal Concept Analysis: Mathematical Foundations. Springer, Berlin-New York 1999.

[GW99b] B. Ganter, R. Wille: Contextual Attribute Logic. In: W. Tepfenhart, W. Cyre (Eds.): Conceptual Structures: Standards and Practices, Springer, Berlin Heidelberg New York 1999, 401-414.

[Ha81] J. Habermas: Theorie kommunikativen Handelns. 2 Bände. Suhrkamp, Frankfurt 1981. 
[He74] H. von Hentig: Magier oder Magister? Über die Einheit der Wissenschaft im Verständigungsprozess. Suhrkamp Verlag, Frankfurt 1974.

[HLSW00] C. Herrmann, P. Luksch, M. Skorsky, R. Wille: Algebras of Semiconcepts and Double Boolean Algebras. Contributions to General Algebra 13, 2000.

[Ka88] I. Kant: Logic. Dover, New York 1988.

[K101a] J. Klinger: Simple Semiconcept Graphs: a Boolean Logic Approach. In: H. S. Delugach, G. Stumme (Eds.): Conceptual Structures: Broadening the Base, Springer Verlag, Berlin-New York 2001, 115-128.

[K101b] J. Klinger: Semiconcept Graphs: Syntax and Semantics, Diplomarbeit, FB Mathematik, TU Darmstadt 2001.

[K102] J. Klinger: Semiconcept Graphs with Variables. In: U. Priss, D. Corbett, G. Angelova (Eds.): Conceptual Structures: Integration and Interfaces, Springer Verlag, Berlin-New York 2002, 382-396.

[KV03] J. Klinger, B. Vormbrock: Contextual Boolean Logic: How did it develop?. FB4-Preprint, TU Darmstadt 2003.

[Pe35] Ch. S. Peirce: Collected Papers. Harvard Uni. Press, Cambridge 1931-35.

[Po01] S. Pollandt: Relational Constructions on Semiconcept Graphs. In: G. Mineau (Ed.): Conceptual Structures: Extracting and Representing Semantics. Dept. of Computer Science. University Laval, Quebec, Canada, 2001, 171-185.

[Po02] S. Pollandt: Relation Graphs - A Structure for Representing Relations in Contextual Logic of Relations. In: U. Priss, D. Corbett, G. Angelova (Eds.): Conceptual Structures: Integration and Interfaces, Springer Verlag, Berlin-New York 2002, 382-396.

[PoW00] S. Pollandt, R. Wille: On the Contextual Logic of Ordinal Data. In: B. Ganter, G. W. Mineau (Eds.): Conceptual Structures: Logical, Linguistic, and Computational Issues, Springer Verlag, Berlin-New York 2000, 249-262.

[Pr98a] S. Prediger: Kontextuelle Urteilslogik mit Begriffsgraphen, Shaker Verlag, Aachen 1998.

[Pr98b] S. Prediger: Simple Concept Graphs: A Logic Approach. In: M.-L. Mugnier, M. Chein (Eds): Conceptual Structures: Theory, Tools and Application, Springer Verlag, Berlin-Heidelberg 1998, 225-239.

[Pr00] S. Prediger: Nested Concept Graphs and Triadic Power Context Families: A Situation-Based Contextual Approach. In: B. Ganter, G. W. Mineau (Eds.): Conceptual Structures: Logical, Linguistic, and Computational Issues, Springer Verlag, Berlin-New York 2000, 249-262.

[PW99] S. Prediger, R. Wille: The lattice of concept graphs of a relationally scaled context. In: W. Tepfenhart, W. Cyre (Eds.): Conceptual Structures: Standards and Practices, Springer, Berlin Heidelberg New York 1999, 401-414.

[Se01] T. B. Seiler: Begreifen und Verstehen: Ein Buch über Begriffe und Bedeutungen. Verlag Allgemeine Wissenschaft, Mühltal 2001.

[SW03] L. Schoolmann, R. Wille: Concept Graphs with Subdivision: a Semantic Approach. FB4-Preprint, TU Darmstadt 2003.

[So84] J. F. Sowa: Conceptual Structures: Information Processing in Mind and Machine. Adison-Wesley, Reading 1984.

[So92] J. F. Sowa: Conceptual Graphs Summary. In: T. E. Nagle, J. A. Nagle, L. L. Gerholz, P. W. Eklund (Eds.): Conceptual Structures: Current Research and Practice. Ellis Horwood, 1992, 3-51.

[Ta00] J. Tappe: Simple Concept Graphs with Universal Quantifiers. In: G. Stumme (Ed.): Working with Conceptual Structures. Contributions to ICCS 2000. Shaker, Achen 2000, 94-108. 
[Wi96] R. Wille: Restructuring Mathematical Logic: An Approach based on Peirce's Pragmatism. In: A. Ursini, P. Agliano (Eds.): Logic and Algebra. Marcel Dekker, New York 1996, 267-281.

[Wi97] R. Wille: Conceptual Graphs and Formal Concept Analysis. In: D. Lukose, H. Delugach, M. Keeler, L. Searle, J. Sowa (eds.): Conceptual Structures: Fullfilling Peirce's Dream. Springer, Berlin-New York 1997, 290-303.

[Wi98] R. Wille: Triadic Concept Graphs. In: M.-L. Mugnier, M. Chein (Eds): Conceptual Structures: Theory, Tools and Application, Springer Verlag, Berlin-New York 1998, 194-208.

[Wi00a] R. Wille: Boolean Concept Logic. In: B. Ganter, G.W. Mineau (Eds.): Conceptual Structures: Logical, Linguistic, and Computational Issues, Springer Verlag, Berlin-New York 2000, 317-331.

[Wi00b] R. Wille: Contextual Logic Summary. In: G. Stumme (Ed.): Working with Conceptual Structures. Contributions to ICCS 2000. Shaker, Aachen 2000, 256276 .

[Wi00c] R. Wille: Lecture Notes on Contextual Logic of Relations. FB4-Preprint, TU Darmstadt 2000.

[Wi01] R. Wille: Boolean Judgment Logic. In: H. S. Delugach, G. Stumme (Eds.): Conceptual Structures: Broadening the Base, Springer Verlag, Berlin-New York 2001, 115-128.

[Wi02a] R. Wille: Existential Graphs of Power Context Families. In: U. Priss, D. Corbett, G. Angelova (Eds.): Conceptual Structures: Integration and Interfaces, Springer Verlag, Berlin-New York 2002, 382-396.

[Wi02b] R. Wille: The Contextual-Logic Structure of Distinctive Judgments. In: U. Priss, D. Corbett, G. Angelova (Eds.): Foundations and Applications of Conceptual Structures - Contributions to ICCS 2002, Bulgarian Academiy of Sciences, 92-101.

[Wi03] R. Wille: Conceptual Content as Information - Basics for Contetxual Judgment Logic. FB4-Preprint, TU Darmstadt 2003. 